\title{
Transcatheter aortic valve implantation (TAVI) in patients with aortic regurgitation
}

\author{
Matthias Thielmann, Konstantinos Tsagakis, Mohamad El Gabry, Heinz Jakob, Daniel Wendt \\ Department of Thoracic and Cardiovascular Surgery, West-German Heart and Vascular Center Essen, University Duisburg-Essen, Essen, Germany \\ Correspondence to: Prof. Matthias Thielmann, MD, PhD. Department of Thoracic and Cardiovascular Surgery, West-German Heart and Vascular \\ Center Essen, University Duisburg-Essen, Hufelandstraße 55, 45122 Essen, Germany. Email: matthias.thielmann@uk-essen.de.
}

Submitted Aug 22, 2017. Accepted for publication Sep 12, 2017.

doi: 10.21037/acs.2017.09.16

View this article at: http://dx.doi.org/10.21037/acs.2017.09.16

\section{Introduction}

Transcatheter aortic valve implantation (TAVI) has become the standard of care for elderly high-risk patients presenting severe and symptomatic aortic valve stenosis, while surgical aortic valve replacement (sAVR) is still considered as the treatment of choice for younger patients and patients with low and intermediate risk profiles $(1,2)$. With more than 100,000 implants worldwide, TAVI has changed the paradigm in the treatment of aortic valve stenosis. The principle concept of TAVI is based on implanting a balloon- or self-expandable transcatheter heart valve into the calcified native aortic annulus (3). Therefore, a certain amount of aortic annulus and/or valve calcification is presumably essential to anchor the valvemounted stent-frame into the annulus. This follows from the fact that, pure, severe aortic regurgitation has been considered as a relative contraindication to TAVI due to the absence of aortic calcification. This is also reflected by the instructions for use of all commercial TAVI systems. On the other hand, there is clearly an unmet clinical need for TAVI as high-risk patients frequently present with a combined aortic valve disease with predominant regurgitation without a reasonable amount of calcium or even pure aortic regurgitation without any aortic valve calcification. Up to now, those patients were mostly treated by sAVR. More recent experiences with TAVI in patients with aortic regurgitation has been reported in several case reports and small clinical studies. The first successful cases were reported as 'off-label' procedures by using the CoreValve ${ }^{\mathrm{TM}}$ (Medtronic, Minneapolis, Minnesota, USA) as well as the Edwards Sapien THV system (Edwards Lifesciences LLC, Irvine, CA, USA) (4-6). Since then, the only approved valve system to be used in pure aortic regurgitation is the JenaValve ${ }^{\mathrm{TM}}$ prosthesis (JenaValve Technology GmbH, Munich, Germany) $(7,8)$. The J-valve ${ }^{\mathrm{TM}}$ system (JC Medical, Inc. Burlingame, CA, USA and Suzhou, China), a recently introduced new, also selfexpandable TAVI-system has also been used in pure aortic regurgitation (9) and just recently, the Lotus ${ }^{\mathrm{TM}}$ valve system (Boston Scientific, Marlborough, MA, USA) was reported to be used successfully for treatment of patients with pure aortic regurgitation. Another interesting concept of a selfexpandable transcatheter valve, the Symetis ACURATE TA $^{\mathrm{TM}}$ device (Symetis S.A., Ecublens, Switzerland) has been introduced firstly for pure aortic regurgitation by our Essen TAVI group (10), being the largest single-center study published so far.

In the following, a case example of a high-risk patient suffering from symptomatic pure aortic regurgitation undergoing a TAVI procedure is presented. This procedure was performed as a Live-case during the Aortic Live 2 Meeting in 2014 at the West-German Heart Center Essen.

\section{Clinical vignette}

A 75-year-old male patient with pure aortic regurgitation grade II-III with dyspnea and reduced functional status NYHA II-III presenting a high-risk for sAVR with a Logistic EuroSCORE of $26.2 \%$ and a STS-score of $11.5 \%$ was referred to our institution. In addition to pure AR, the patient had a three-vessel coronary artery disease and reduced LV-function with prior CABG surgery in 1989 and a recent PCI before TAVI. In preparation of the TAVI procedure a coronary angiography as well as a TOE and a 

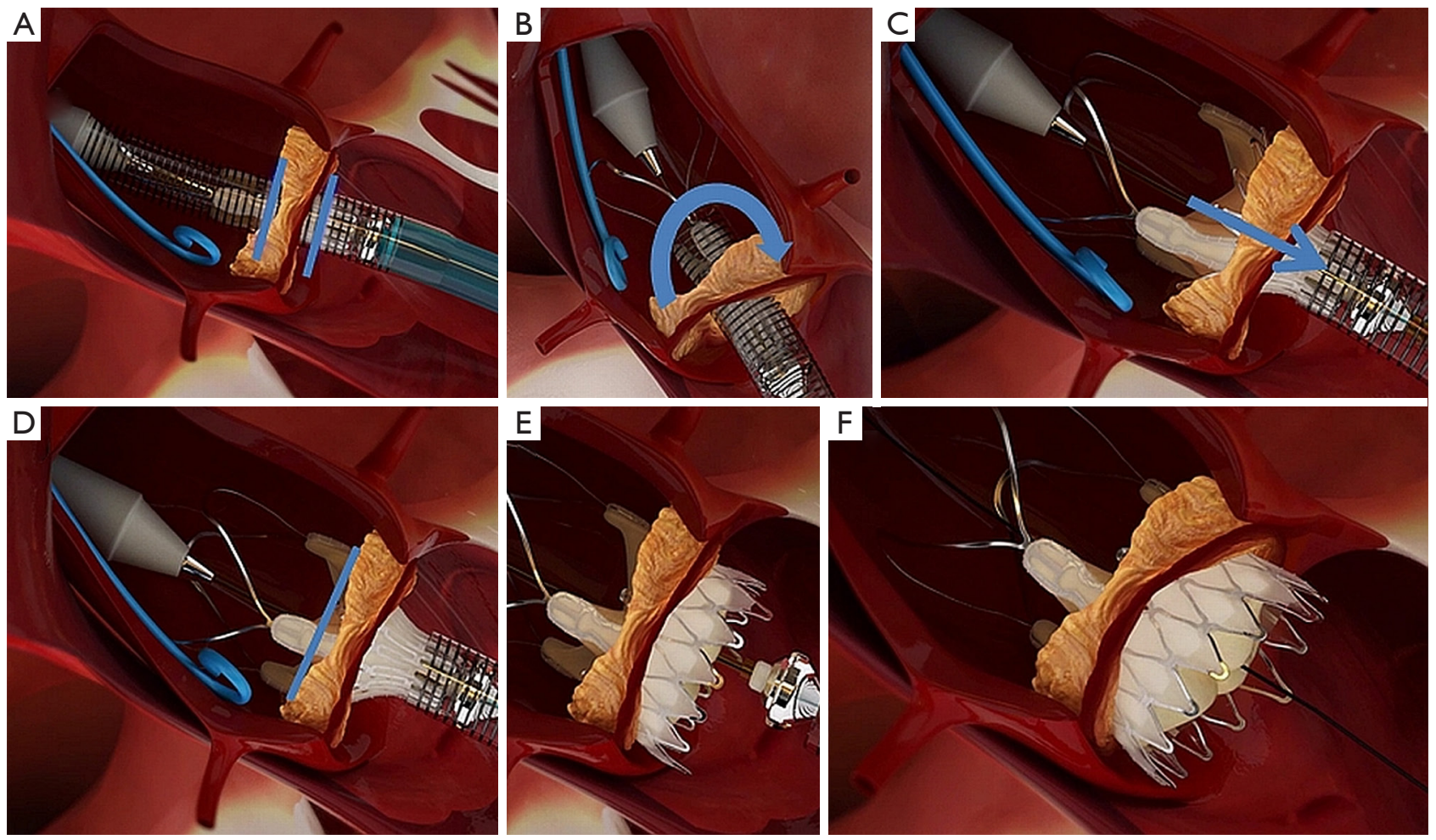

Figure 1 With this figure, the sequence of implantation steps using the Symetis ACURATE TA system are shown. (A) Intra-annular positioning of the device (blue bars indicating the annular level); (B) anatomical correct rotation (blue arrow) of the device; (C) partial unsheathing and pulling of the device (blue arrow) and placement of the upper crown; (D) after correct placement of the upper crown the lower crown $(\mathrm{E})$ is released with the valve self-aligned within the aortic annulus $(\mathrm{F})$.

CT for aortic valve sizing was performed. The indication for a TAVI procedure using a Symetis ACURATE TA system via the transapical approach was made by the Essen TAVI team (Figure 1).

\section{Comments}

To date, TAVI has been suggested as an alternative treatment option to sAVR in selected patients and has become a widely practiced and accepted therapeutic option $(1,2)$. TAVI was primarily developed for patients presenting with aortic valve stenosis; however, although aortic stenosis is more prevalent, also high-risk patients presenting with aortic regurgitation may benefit from such novel catheter-based techniques. The original concept of TAVI was based on balloon-expandable valve, which by all means depends on aortic annulus calcification. Contrarily, self-expandable valves offer high and permanent recoil forces and therefore this concept is better suited to treat aortic regurgitation. So far, the JenaValve ${ }^{\mathrm{TM}}$ technology is the only TAVI-device which has received extended CE-mark approval for the treatment of aortic regurgitation. We decided to use and evaluate the Symetis ACURATE ${ }^{\mathrm{TM}}$ prosthesis in patients presenting with aortic regurgitation, as we tested this system in an acute animal study. Within this evaluation, by nature, all animals presented without any calcification and with normal aortic valves. Even in this setting, we observed no valve dislocation and a safe fixation in the native, non-calcified aortic annulus (11). Some of the above-presented concepts offer tactile feedback, as they are implanted through the apex of the left ventricle, and, they come with a unique feature of self-positioning of self-aligning. Therefore, even in aortic regurgitation, these technologies offering tactile feedback guides the operator for optimal deployment. In addition to this beneficial feature, some devices offer a partial re-sheathing or repositioning of the valve until final deployment.

\section{Conclusions}

In conclusion, the presented TAVI case successfully 
demonstrates the feasibility of the Symetis ACURATE-TA system in a 75-year-old high-risk candidate suffering from symptomatic and pure aortic valve regurgitation. Except the Jena-Vale system, several self-expandable transcatheter aortic valve devices are currently used as 'off-labelprocedures' for treatment of pure aortic valve regurgitation. The majority of currently published papers show feasibility of TAVI using several THV devices in patients with pure aortic regurgitation with low intra-procedural complication rates. Nevertheless, mid-term results are debatable and currently only available for some small series. In the future, the experience with TAVI in patients presenting with pure aortic regurgitation may increase. Therefore, there are currently some strong ambitions to harmonize and collect the results of the use of TAVI in pure aortic regurgitation in large worldwide registries.

\section{Acknowledgements}

None.

\section{Footnote}

Conflicts of Interest: The authors have no conflicts of interest to declare.

\section{References}

1. Leon MB, Smith CR, Mack M, et al. Transcatheter aortic-valve implantation for aortic stenosis in patients who cannot undergo surgery. N Engl J Med 2010;363:1597-607.

2. Smith CR, Leon MB, Mack MJ, et al. Transcatheter versus surgical aortic-valve replacement in high-risk patients. $\mathrm{N}$ Engl J Med 2011;364:2187-98.

3. Cribier A, Eltchaninoff $\mathrm{H}$, Bash A, et al. Percutaneous

Cite this article as: Thielmann M, Tsagakis K, El Gabry M, Jakob H, Wendt D. Transcatheter aortic valve implantation (TAVI) in patients with aortic regurgitation. Ann Cardiothorac Surg 2017;6(5):558-560. doi: 10.21037/acs.2017.09.16 transcatheter implantation of an aortic valve prosthesis for calcific aortic stenosis: first human case description. Circulation 2002;106:3006-8.

4. D'Ancona G, Pasic M, Buz S, et al. TAVI for pure aortic valve insufficiency in a patient with a left ventricular assist device. Ann Thorac Surg 2012;93:e89-91.

5. Roy DA, Schaefer U, Guetta V, et al. Transcatheter aortic valve implantation for pure severe native aortic valve regurgitation. J Am Coll Cardiol 2013;61:1577-84.

6. Hildebrandt HA, Erbel R, Kahlert P. Compassionate use of the self-expandable medtronic CoreValve prosthesis for the treatment of pure aortic regurgitation in a patient at prohibitive risk for surgical valve replacement. Catheter Cardiovasc Interv 2013;82:E939-43.

7. Seiffert M, Diemert P, Koschyk D, et al. Transapical implantation of a second-generation transcatheter heart valve in patients with noncalcified aortic regurgitation. JACC Cardiovasc Interv 2013;6:590-7.

8. Seiffert M, Bader R, Kappert U, et al. Initial German experience with transapical implantation of a secondgeneration transcatheter heart valve for the treatment of aortic regurgitation. JACC Cardiovasc Interv 2014;7:1168-74.

9. Wei L, Liu H, Zhu L, et al. A New Transcatheter Aortic Valve Replacement System for Predominant Aortic Regurgitation Implantation of the J-Valve and Early Outcome. JACC Cardiovasc Interv 2015;8:1831-41.

10. Wendt D, Kahlert P, Pasa S, et al. Transapical transcatheter aortic valve for severe aortic regurgitation: expanding the limits. JACC Cardiovasc Interv 2014;7:1159-67.

11. Wendt D, Pasa S, Kahlert P, et al. A new self-expandable transcatheter aortic valve for transapical implantation: feasibility in acute and chronic animal experiments. Scand Cardiovasc J 2013;47:145-53. 